\title{
Statistics of reaction progress variable and mixture fraction gradients of a pulverised coal jet flame using Direct Numerical Simulation data
}

\author{
Umair Ahmed ${ }^{\mathrm{a}, *}$, Charles Turquand d'Auzay ${ }^{\mathrm{a}}$, Masaya Muto ${ }^{\mathrm{b}}$, Nilanjan \\ Chakraborty $^{\mathrm{a}}$, Ryoichi Kurose ${ }^{\mathrm{b}}$ \\ ${ }^{a}$ School of Engineering, Newcastle University, Newcastle-Upon-Tyne NE1 7RU, United \\ Kingdom \\ ${ }^{b}$ Department of Mechanical Engineering and Science, Kyoto University, Kyoto \\ daigaku-Katsura, Nishikyo-ku, Kyoto 615-8540, Japan
}

\section{Abstract}

The statistical behaviour of the variances, covariance and gradients of the reaction progress variable $(c)$, and the mixture fraction $(\xi)$ have been analysed in a pulverised coal jet flame using a three-dimensional carrier phase direct numerical simulation (DNS) dataset. It has been observed that the Favre-PDFs of $c$ and $\xi$ can be parametrised by the standard $\beta$ function. Furthermore, the log-normal distribution has been found to accurately represent $|\nabla c|$ and $|\nabla \xi|$. It is also found that $\nabla c$ and $\nabla \xi$ remain aligned throughout the flame brush. Finally the joint PDF of $|\nabla c|$ and $|\nabla \xi|$ has been compared with the product of the PDF of $|\nabla c|$ and PDF of $|\nabla \xi|$ extracted from carrier phase DNS, and it has been found that $|\nabla c|$ and $|\nabla \xi|$ are not statistically independent in the case investigated.The bivariate log-normal distributions with and without correlation have also been considered, and the former is

\footnotetext{
*Corresponding author:

Email address: umair.ahmed@newcastle.ac.uk (Umair Ahmed)
} 
found to be in better agreement with the carrier phase DNS data.

Keywords:

Coal combustion, Progress variable, Probability density function, Scalar

gradient statistics, Scalar dissipation

\section{Introduction}

Coal is one of the most abundant fossil fuels, and is expected to play a dominant role in power generation in the foreseeable future due to its low cost and stable supply. However, coal-fired power plants produce large amount of pollutants, hence it has become important to reduce the environmental impact of coal combustion. Carbon Capture and Storage (CCS) can significantly reduce the emissions from coal fired power plants, but this reduction in emissions is usually achieved at the cost of plant efficiency. Hence, the design of a more efficient power plant requires knowledge of the physical and chemical mechanisms governing pulverised coal combustion. Computational fluid dynamics (CFD) can play an important role in this process, as it can provide details of the flow field and its interaction with chemical mechanism which otherwise are not available from experiments.

Recently, CFD simulations have been performed for pulverised coal combustion using the Reynolds Averaged Navier-Stokes (RANS) [1] and Large Eddy Simulation (LES) [2-6] frameworks. These approaches are computationally less expensive, but require closures of several quantities appearing in the governing equations. Many recent studies performed using RANS [1] and LES $[2,5]$ techniques rely on the models developed for gaseous phase combustion, which may potentially lead to erroneous predictions of the pollutants. 
Direct Numerical Simulation (DNS) for the carrier gaseous phase can play an important role in providing a detailed physical understanding of the fluid mechanics and chemical processes involved in pulverised coal combustion, as it does not require any modelling for turbulence and turbulence-chemistry interaction. Furthermore, carrier phase DNS data of pulverised coal combustion can also be used to inform closure models needed in RANS and LES simulations. Recently, DNS has made significant contributions to the physical understanding and modelling of spray and gaseous phase combustion [7-9] using a detailed analysis of the behaviour of progress variable and mixture fraction gradients.

The existing relations between the mixture fraction $(\xi)$ and the progress variable $(c)$ can play an important role in the flamelet modelling of pulverised coal combustion $[2,6,10]$. The statistics of $c$ and $\xi$ (i.e. PDFs and joint PDFs) are essential for the development of flamelet models [11, 12]. Understanding of the statistical behaviour of $c$ and $\xi$ gradients plays a pivotal role in the modelling of (cross-) scalar dissipation rates, which subsequently contribute to the closure of the mean reaction rate. The (cross-) scalar dissipation rate can be defined as :

$$
N_{\Lambda_{i} \Lambda_{j}}=\alpha\left|\nabla \Lambda_{i}\right|\left|\nabla \Lambda_{j}\right| \cos \left(\theta_{\Lambda_{i} \Lambda_{j}}\right),
$$

where $\Lambda_{i}$ represents the scalar and $\alpha$ is the diffusivity of the scalar.

In many recent studies a presumed PDF approach is adopted $[4,5]$, but the statistics of $c$ and $\xi$ and their respective gradients, which are essential for this modelling approach, have received relatively limited attention. Hence, understanding statistical behaviours of $|\nabla c|$ and $|\nabla \xi|$ are of fundamental importance for closing the mean/filtered reaction rate. In this work the 
statistical behaviours of $c, \xi$ and their respective gradients are investigated using a carrier phase DNS dataset of a pulverised coal jet flame [13].

The paper is organised as follows: the next section provides a description of the DNS data used. The results are presented and discussed in section 3 and the conclusions are summarised in the last section.

\section{DNS data}

The carrier phase direct numerical simulation (DNS) data of a pulverised coal jet flame performed by Hara et al. [13] has been used in this study. This DNS dataset has been validated by comparing the results with the experimental data of Hwang et al. [14]. Note that in this experiment the flame is supported by a gas flame and has a negligible char-burnout [14].The computational domain for the DNS consists of a rectangular domain of size $500 \mathrm{~mm} \times 1000 \mathrm{~mm} \times 500 \mathrm{~mm}$, which is descretised by a Cartesian $320 \times 1632 \times$ 320 non-uniform staggered grid. The flow enters the domain through an annular pipe in the $y$ direction with a central pipe of $6 \mathrm{~mm}$ inner diameter $(D)$ and also through a surrounding annulus of $8 \mathrm{~mm}$ inner diameter $(d)$. Air and coal particles are injected through the central pipe at a velocity of $6.37 \mathrm{~m} / \mathrm{s}$ while $\mathrm{CH}_{4}$ is injected from the surrounding annulus at a velocity of $1.98 \mathrm{~m} / \mathrm{s}$. A streamwise velocity of $0.25 \mathrm{~m} / \mathrm{s}$ is assigned to the surrounding air. A schematic of the flow configuration is presented in Fig. 1a. For this DNS analysis Newlands coal has been considered and its properties have been provided elsewhere $[13,14]$, and thus are not repeated here.

A modified two-step chemical mechanism proposed by Franzelli et al. [15] is used to account for the volatile matter combustion. The standard 
mass, momentum, energy and species transport equations for reacting flows are solved for the Eulerian gaseous phase. The individual coal particles are tracked in a Lagrangian sense by solving the transport equations of position, velocity, temperature and mass. These equations account for heat transfer between the gas and dispersed coal phase, the heat transfer due to devolatilisation, and water evaporation and the mass of chemical species in volatile matter in coal [13]. It is assumed that the shape of the coal particle remains spherical, and collision and breakup of particles are not considered [13]. It is also assumed that the change in particle mass leads only to change in density while the particle diameter remains unchanged, and the influence of gravity on the particles is not considered [13]. Further details of the numerical set-up can be found in [13]. The simulation has been performed using the code known as $\mathrm{FK}^{3}[13,16-18]$. The coupling between Eulerian and Lagrangian phases is achieved via the Particle-Source-In-Cell approach [13]. A Rosin-Rammler distribution with a number-averaged diameter of $25 \mu \mathrm{m}$ (and a mass-averaged diameter of $33 \mu \mathrm{m}$ ) is adopted as the coal particle diameter distribution. Further details on the boundary conditions and grid quality can be found in [13].

In the post-processing of the carrier phase DNS data, the time (Reynolds) averaged quantities (denoted by $\bar{\lambda}$ ), Favre averaged quantities (denoted by $\widetilde{\lambda}=\overline{\rho \lambda} / \bar{\rho}$ ) and Favre fluctuations (denoted by $\lambda^{\prime \prime}=\lambda-\widetilde{\lambda}$ ) have been computed in time over several instantaneous realisations of the flow field after the initial transients have decayed. The statistical convergence has been ensured by establishing that halving the number of instantaneous samples used for obtaining the statistics did not have any noticeable difference (the maximum 
difference in the mean values of $|\nabla c|$ and $|\nabla \xi|$ remains less than 1\%). The absence of gravity in the DNS calculation may influence the statistics obtained in the outer region of the jet in the radial direction as explained by Hara et al [13]; hence the outer radial region of the jet is not included in the current analysis.

\section{Results and discussion}

\subsection{Flame turbulence interaction}

Figure 1 shows the instantaneous gradients of the progress variable $(c)$ (where $c=\left(Y_{c}-Y(\xi)_{c_{\min }}\right) /\left(Y(\xi)_{c_{\max }}-Y(\xi)_{c_{\min }}\right.$ ), where $Y_{c}=Y_{\mathrm{CO}_{2}}+Y_{\mathrm{H}_{2} \mathrm{O}}$ ) $[11,19,20]$, and the mixture fraction $(\xi)$ (defined using Bilger's definition as $\xi=\left(\beta-\beta_{O}\right) /\left(\beta_{f}-\beta_{O}\right)[21]$, where $\beta=2 Y_{C} / W_{C}+0.5 Y_{H} / W_{H}-Y_{O} / W_{O}, \beta_{f}=$ $(2 a+0.5 b-c) / W_{C_{a} H_{b} O_{c}}$ and $\beta_{O}=-Y_{O^{\infty}} / W_{O}$, with $Y_{O_{\infty}}$ being the elemental oxygen mass fraction in the pure oxidiser stream; $Y_{M}$ and $W_{M}$ are the mass fraction and molecular mass of the element/species $M$, respectively). Note that the definition of $\xi$ uses elemental mass fractions and can in principle be used for multiple fuel sources. In the reaction progress variable definition, $Y(\xi)_{c_{\min }}$ and $Y(\xi)_{c_{\max }}$ are the sum of $Y_{\mathrm{CO}_{2}}$ and $Y_{\mathrm{H}_{2} \mathrm{O}}$ in the unburnt mixture for the local value of $\xi$ and in the fully burnt products according to the equilibrium solution for the local value of mixture fraction $\xi$, respectively. It can be seen in Figs. 1b and 1c that the gradients of $c$ and $\xi$ are enhanced by the turbulence-shear interaction. An increase in the mass fraction of volatile matter in the downstream direction can be seen in Fig. 1d, which is obtained as a result of the devolatilisation from the coal particles far downstream of the jet exit. 


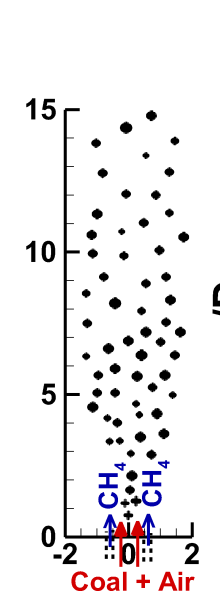

(a)

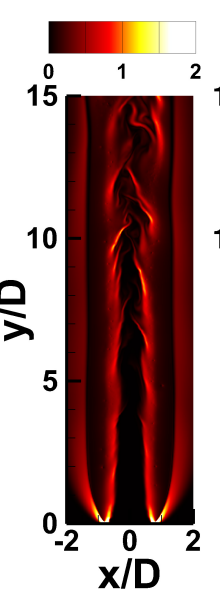

(b)

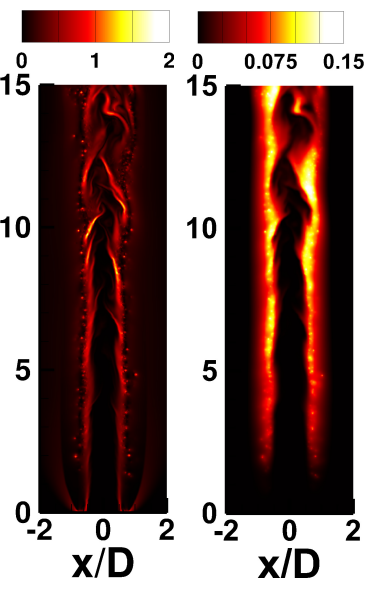

(c)

(d)

Figure 1: (a) Schematic diagram of the flow field. Instantaneous (b) $|\nabla c| \times D$, (c) $|\nabla \xi| \times D$, (d) mass fraction of volatile matter at the $y-x$ midplane

The influence of turbulence on the flame can be further investigated by examining the variance of the progress variable $\left(\widetilde{c^{\prime \prime}}\right)$, variance of the mixture fraction $\left(\widetilde{\xi^{\prime \prime}}\right)$ and the co-variance of the progress variable and mixture fraction $\left(\widetilde{c^{\prime \prime} \xi^{\prime \prime}}\right)$. Understanding of these quantities is essential for improved scalar dissipation and reaction rate closures in the context of RANS and LES frameworks. Figure 2 shows the variations of $\widetilde{c^{\prime \prime}}, \widetilde{\xi^{\prime \prime}}$ and $\widetilde{c^{\prime \prime} \xi^{\prime \prime}}$ at different downstream locations of the jet exit. The values of $\widetilde{c^{\prime \prime}}$ obtained from DNS data are compared with the Bray Moss Libby (BML) closure [22] $\widetilde{c^{\prime \prime 2}}=\widetilde{c}(1-\widetilde{c})+\mathcal{O}(\gamma)$, where $\gamma$ represents the burning mode PDF and is negligible in high Damköhler number flames. The difference between $\widetilde{c}(1-\widetilde{c})$ and $\widetilde{c^{\prime \prime 2}}$ provides a measure of the departure from an asymptotically infinitely fast chemistry limit and a greater difference is obtained for smaller values of $D a$ (i.e. provides a measure of chemical time scale compared with the tur- 
bulent mixing time scale). It can be seen from Fig. 2 that the value of $\widetilde{c^{\prime \prime 2}}$ is much smaller than the BML limit and increases with the distance away from the jet exit. The low values of $\widetilde{c^{\prime \prime 2}}$ imply that the contributions from the burning mode PDF are non-negligible [23]. The increase in the magnitude of $\widetilde{c^{\prime \prime 2}}$ with the distance away from the jet exit can be explained by an increase in volatile gas mass fraction which leads to the availability of more reactive flammable mixture with relatively smaller chemical time scale. The characteristic Damköhler number increases in the downstream direction which is reflected in the increase in the value of the variance of reaction progress variable $\widetilde{c^{\prime \prime 2}}$.

An increase in the value of $\widetilde{\xi^{\prime \prime 2}}$ can be seen with an increase in $y / D$ in Fig. 2. This is due to enhanced straining caused by turbulent fluctuations and devolatilisation of coal particles. In Fig. 2, the co-variance $\widetilde{c^{\prime \prime} \xi^{\prime \prime}}$ shows mostly positive values throughout the flame brush but some negative values can be discerned towards the burned gas side of the flame brush at $y / D=12.5$. It is important to understand the behaviour of the correlation between $c$ and $\xi$ in order to explain the statistical behaviour of the co-variance $c^{\prime \prime} \xi^{\prime \prime}$, and will be addressed in the next sub-section. Ribert et al. [24] proposed a model in the context of the Libby-Williams [25] model for partially premixed flames for the co-variance of fuel mass fraction and mixture fraction (i.e. $\left.\left.\widetilde{Y_{F}^{\prime \prime} \xi^{\prime \prime}}=\widetilde{\left(Y_{F}^{\prime \prime 2}\right.} \widetilde{\xi^{\prime \prime}}\right)^{1 / 2}\right)$. The same modelling philosophy $\widetilde{c^{\prime \prime} \xi^{\prime \prime}}=\left(\widetilde{c^{\prime \prime}} \widetilde{\xi^{\prime \prime 2}}\right)^{1 / 2}$ has been tested and a good agreement is seen for $0<\widetilde{c}<0.8$, but the model fails to capture the negative trends observed in $\widetilde{c^{\prime \prime} \xi^{\prime \prime}}$ in the current flame.

It can be observed in Fig. 2 that the Reynolds number of the flame being investigated is relatively low. In the light of several previous DNS 

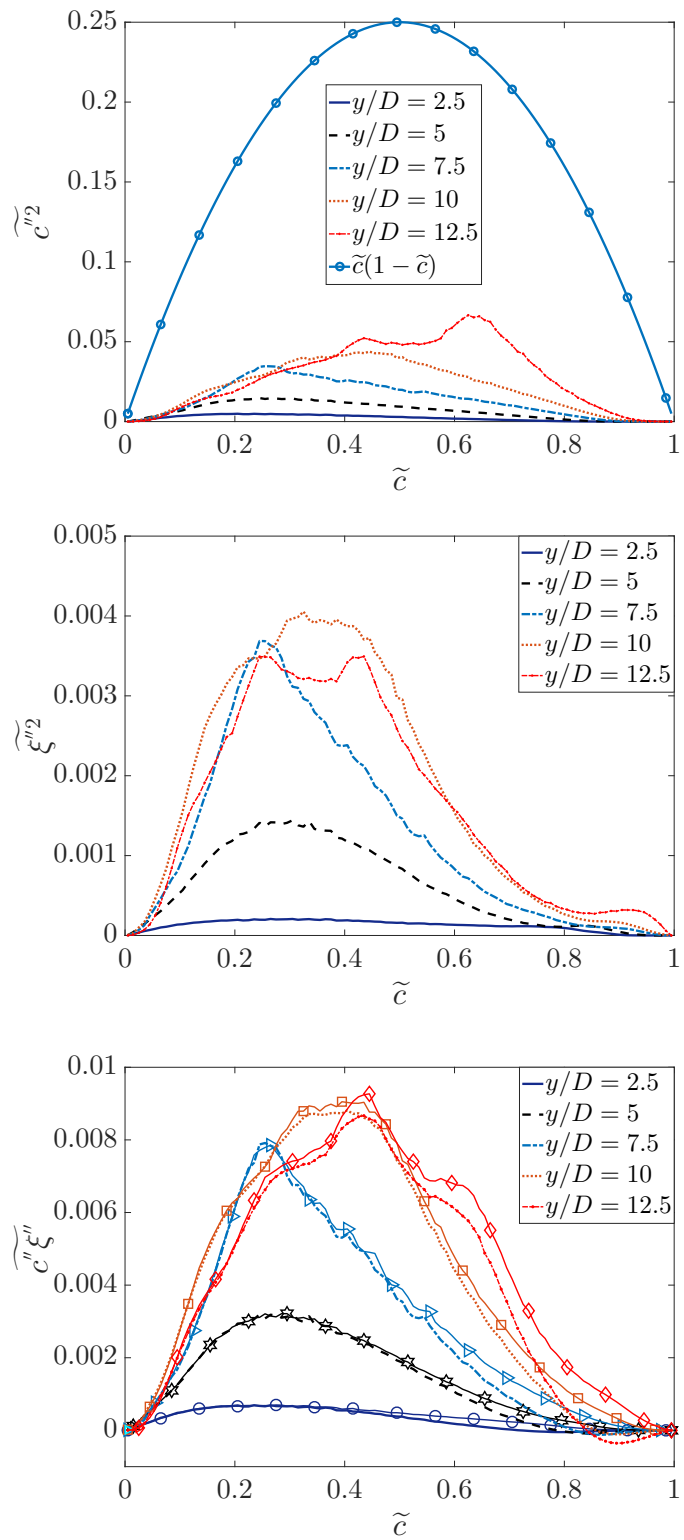

Figure 2: Variations of $\widetilde{c^{\prime \prime 2}}, \widetilde{\xi^{\prime \prime 2}}$ and $\widetilde{c^{\prime \prime} \xi^{\prime \prime}}$ across the flame brush at different locations. Lines with symbols in the figure for $\widetilde{c^{\prime \prime} \xi^{\prime \prime}}$ show the results from the model of Ribert et al. [24]. 
analyses on single-phase gaseous flames with moderate Reynolds numbers [7, 26-29] it is argued here that the results obtained from this flame would be valid for higher Reynolds numbers. Furthermore, it should be noted that the models developed in the aforementioned gaseous flame DNS studies have performed well for simulating laboratory scale burners with much higher Reynolds numbers [30-34].

\subsection{Behaviour and modelling of the PDFs of $c$ and $\xi$}

In order to close the Favre-averaged scalar and cross-scalar dissipation rates (in the context of RANS $\widetilde{N}_{\Lambda_{1} \Lambda_{2}} \approx \widetilde{\epsilon}_{\Lambda_{1} \Lambda_{2}}=\overline{\rho \alpha \nabla \Lambda_{1}^{\prime \prime} \Lambda_{2}^{\prime \prime}} / \bar{\rho}$ ), the variance and co-variance are needed according to the linear relaxation models $\widetilde{\epsilon}_{\Lambda_{1} \Lambda_{2}}=$ $C_{\Lambda_{1} \Lambda_{2}}(\widetilde{\epsilon} / \widetilde{k}) \widetilde{\Lambda_{1}^{\prime \prime} \Lambda_{2}^{\prime \prime}}$ where $C_{\Lambda_{1} \Lambda_{2}}$ is the model parameter and $\widetilde{k}$ and $\widetilde{\epsilon}$ are the turbulent kinetic energy and its dissipation respectively $[8,35]$. The variance and cross-variance closures require the knowledge of the joint PDF of $c$ and $\xi$ (i.e. $P(c, \xi)$ ) and Favre PDFs of $c$ and $\xi$ (i.e. $(\widetilde{P}(c))$ and $\widetilde{P}(\xi)$ ), as $\widetilde{c^{\prime \prime 2}}=$ $\int_{0}^{1}(c-\widetilde{c})^{2} \widetilde{P}(c) \mathrm{d} c, \widetilde{\xi^{\prime \prime}}=\int_{0}^{1}(\xi-\widetilde{\xi})^{2} \widetilde{P}(\xi) \mathrm{d} \xi$ and $\widetilde{c^{\prime \prime} \xi^{\prime \prime}}=(1 / \bar{\rho}) \int_{0}^{1} \int_{0}^{1} \rho(c-\widetilde{c})(\xi-$ $\widetilde{\xi}) P(c, \xi) \mathrm{d} c \mathrm{~d} \xi[9]$, where $\widetilde{P}(c)=\left.\rho\right|_{c} P(c) / \bar{\rho}$ and $\left.\rho\right|_{c}$ is the mean gas density conditioned on $c$ and $P(c)$ is the marginal PDF of $c$.

Figure 3 shows the joint PDFs of $c$ and $\xi$ for different values of $\widetilde{c}$ at $y / D=7.5$, which shows a positive correlation between $c$ and $\xi$ towards the unburned gas side of the flame brush but this positive correlation weakens with increasing $c$. However, this correlation between $c$ and $\xi$ becomes negative towards the burned gas side. The same qualitative behaviour has been observed at other axial locations but the most probable value of $c$ increases with an increase in the axial distance from the jet exit (not shown here). The probability of finding devolatilised fuel is relatively small close to the jet exit 
(see Fig. 1d), and the availability of fuel increases with height which provides higher probability of flammable mixture due to devolatilisation; thus the chemical reaction rate advances rapidly in this region. This is reflected in the increased probability of finding high values of $c$ in the downstream direction as shown in Fig. 3. The positive correlation between $c$ and $\xi$, as shown in Fig. 3, is responsible for predominantly positive values of covariance $\left(\widetilde{c^{\prime \prime} \xi^{\prime \prime}}\right)$ for the major part of the flame brush. However, the negative correlation between $c$ and $\xi$ towards the burned gas side of the flame brush yields negative values of $\widetilde{c^{\prime \prime} \xi^{\prime \prime}}$. The fuel-mixture with $\xi / \xi_{s t} \approx 1$ is more reactive than the fuel-lean mixtures (i.e. $\xi / \xi_{s t}<1$ ), and thus the probability of finding high values of $c$ increases with increasing $\xi$ for $\xi / \xi_{s t}<1$. As high values of $c$ are obtained for $\xi / \xi_{s t} \approx 1$, a negative correlation branch can be seen for $\xi / \xi_{s t}>1$, which is responsible for negative values of $c^{\prime \prime} \xi^{\prime \prime}$ towards the burned gas side of the flame brush, and weakening of positive correlation of $c$ and $\xi$ at the middle of the flame brush.

Figure 4 shows the Favre PDFs of $c$ and $\xi$ at $y / D=2.5$ and $y / D=10$. At both sampling locations similar qualitative behaviours have been observed for both $c$ and $\xi$. Distinct peaks at $c=0, c=0.5$ and $c=0.85$ can be seen for the Favre PDF of $c$. The peak around $c=0.85$ moves towards $c=1$ as the distance from the jet exit increases. This implies that there is incomplete burning near the jet exit due to the lack of volatile matter in the reacting mixture. The Favre PDF of $c$ can be approximated by the modified $\beta$-PDF $\left(\beta_{m}-\mathrm{PDF}[36-38]\right)$ parametrised in terms of mean and variance as :

$$
\beta_{m}\left(\Lambda, a^{\prime}, b^{\prime}\right)=\quad \begin{aligned}
& \frac{\Gamma\left(a^{\prime}+b^{\prime}\right)}{\Gamma\left(a^{\prime}\right) \Gamma\left(b^{\prime}\right)}\left(\Lambda_{\max }-\Lambda_{\min }\right)^{1-a^{\prime}-b^{\prime}} \\
& \left(\Lambda-\Lambda_{\min }\right)^{a^{\prime}-1}\left(\Lambda_{\max }-\Lambda\right)^{b^{\prime}-1},
\end{aligned}
$$



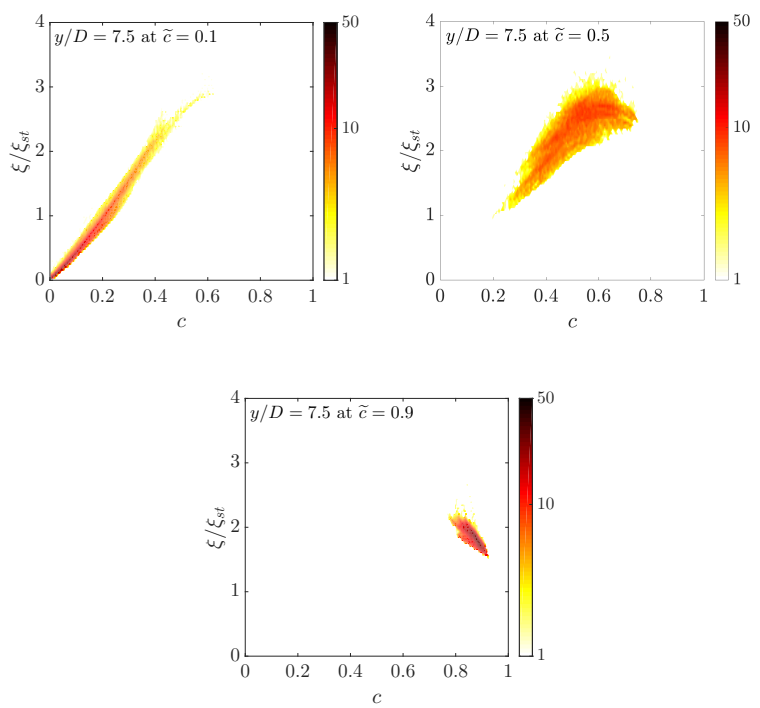

Figure 3: Joint PDFs of $c$ and $\xi$ at $y / D=7.5$ for different iso-surfaces of $\widetilde{c}$.

where the coefficients $a^{\prime}$ and $b^{\prime}$ definitions are:

$$
\begin{aligned}
& a^{\prime}=\frac{\widetilde{\Lambda}-\Lambda_{\min }}{\Lambda_{\max }-\Lambda_{\min }}\left[\frac{\left(\widetilde{\Lambda}-\Lambda_{\min }\right)\left(\Lambda_{\max }-\widetilde{\Lambda}\right)}{\widetilde{\Lambda^{\prime \prime}}}-1\right], \\
& b^{\prime}=\frac{\Lambda_{\max }-\widetilde{\Lambda}}{\Lambda_{\max }-\Lambda_{\min }}\left[\frac{\left(\widetilde{\Lambda}-\Lambda_{\min }\right)\left(\Lambda_{\max }-\widetilde{\Lambda}\right)}{\widetilde{\Lambda^{\prime \prime}}}-1\right],
\end{aligned}
$$

where $\Lambda$ is a scalar, $\Gamma$ is the gamma function defined by $\Gamma(z)=\int_{0}^{\infty} x^{z-1} e^{-x} d x$. Note that by setting $\Lambda_{\max }=1.0$ and $\Lambda_{\min }=0.0$, the standard $\beta$-PDF is recovered. Figure 4 shows that the standard $\beta$ function satisfactorily captures the DNS trends.

The Favre PDFs of $\xi$ in Fig. 4 show a monomodal behaviour with distinct peaks at $\xi / \xi_{s t} \approx 0$, which remains approximately constant at all axial locations examined. While another peak at $1.5<\xi / \xi_{s t}<2$ can be seen, which decreases with the axial distance. This occurs due to the devolatilisation of 

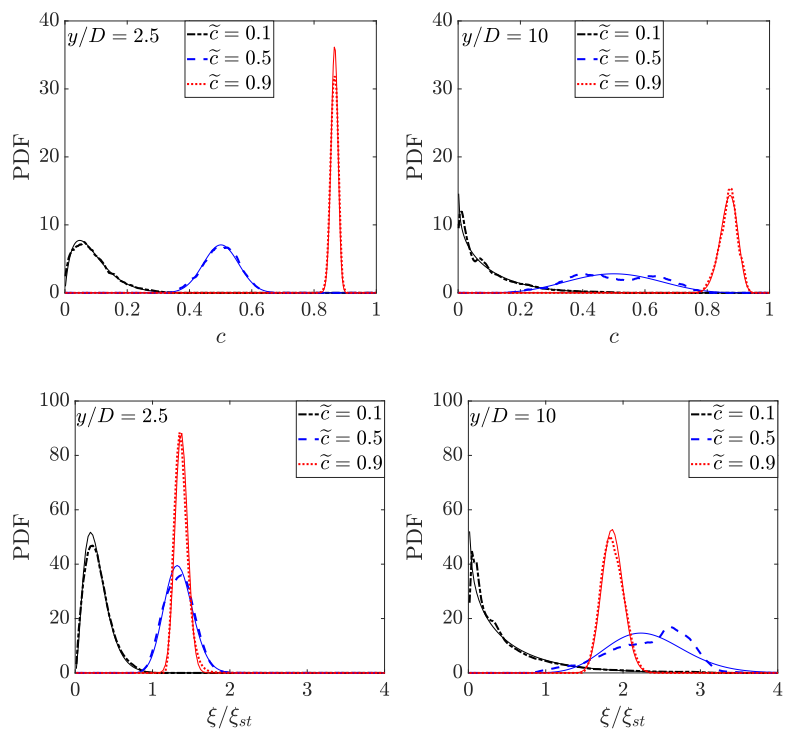

Figure 4: Favre PDFs of $c$ and $\xi$ at different locations within the flame. Solid lines represent $\beta$-function approximations.

coal particles and also due to increased mixing in the downstream direction. In this case a standard $\beta$-PDF is able to capture the $\mathrm{PDF}$ of $\xi$ extracted from the DNS data. This is consistent with the earlier findings for turbulent spray flames [37, 38]. Several other studies for multiphase flows [37], and spray combustion [38, 39] have shown that under certain conditions the standard $\beta$-function is unable to capture the PDF of $\xi$; thus Eq. (2)-(4) need to be used, provided that $\Lambda_{\min }$ and $\Lambda_{\max }$ are carefully chosen to avoid negative values of $a^{\prime}$ and $b^{\prime}$. The issue related to the performance of the standard $\beta$-PDF can also occur in the case of coal combustion and caution must be exercised in the choice of PDF for coal combustion. 


\subsection{Behaviour and modelling of the PDFs of $|\nabla c|$ and $|\nabla \xi|$}

The closures for (cross-) scalar dissipation of $c$ and $\xi$ require an understanding of the gradients of $c$ and $\xi$ as:

$$
\begin{aligned}
\tilde{\epsilon}_{\Lambda_{1} \Lambda_{2}} \approx \quad \tilde{N}_{\Lambda_{1} \Lambda_{2}}= & \frac{1}{\bar{\rho}} \int_{0}^{\infty} \int_{0}^{\infty} \rho \alpha\left|\nabla \Lambda_{1}\right|\left|\nabla \Lambda_{2}\right| \cos \theta_{\Lambda_{1} \Lambda_{2}} \\
& P\left(\left|\nabla \Lambda_{1}\right|,\left|\nabla \Lambda_{1}\right|\right) \mathrm{d}\left|\nabla \Lambda_{1}\right| \mathrm{d}\left|\nabla \Lambda_{2}\right|,
\end{aligned}
$$

as $\widetilde{\epsilon}_{\Lambda_{1} \Lambda_{2}}>>\nabla \nabla \widetilde{\Lambda}_{1} \cdot \nabla \widetilde{\Lambda}_{2}$ in the context of RANS. Figure 5 shows the PDFs of normalised $|\nabla c|$ and $|\nabla \xi|$ at $y / D=2.5$ and $y / D=10$ for different values of $\widetilde{c}$. The PDFs for $|\nabla c|$ and $|\nabla \xi|$ can be approximated by a log-normal distribution for a scalar $\Lambda$, parametrised by the log-normal mean $(\mu)$ and standard deviation $(\sigma)$ as :

$$
f_{\Lambda}(\mu, \sigma)=\frac{1}{\Lambda \sigma \sqrt{2 \pi}} \exp \left[-\frac{(\ln (\Lambda)-\mu)^{2}}{2 \sigma^{2}}\right],
$$

where $\mu=\ln \left[\widetilde{\Lambda}\left(1+\widetilde{\Lambda^{\prime \prime 2}} / \widetilde{\Lambda}\right)^{-1 / 2}\right]$ and $\sigma^{2}=\ln \left[1+\widetilde{\Lambda^{\prime \prime} 2} / \widetilde{\Lambda}\right]$. It can be seen from Fig. 5 that the PDFs of $|\nabla c|$ and $|\nabla \xi|$ are well approximated by the lognormal distribution, but considerable discrepancies between the log-normal distribution and PDFs obtained from DNS data can been observed for small values of $|\nabla c|$ and $|\nabla \xi|$. This behaviour is consistent with the earlier experimental $[40,41]$ and numerical investigations [8, 42, 43] of non-premixed and stratified combustion and also for passive scalar mixing. In the specific case of coal combustion it is argued here that this discrepancy can be attributed to the small fluctuations caused by the interaction of the coal particles in the centre of the jet. Note that despite some shortfalls in the log-normal approximation it can be used for modelling PDFs of $|\nabla c|$ and $|\nabla \xi|$ and consequently also for the closures of the scalar dissipation rates of $c$ and $\xi$. 

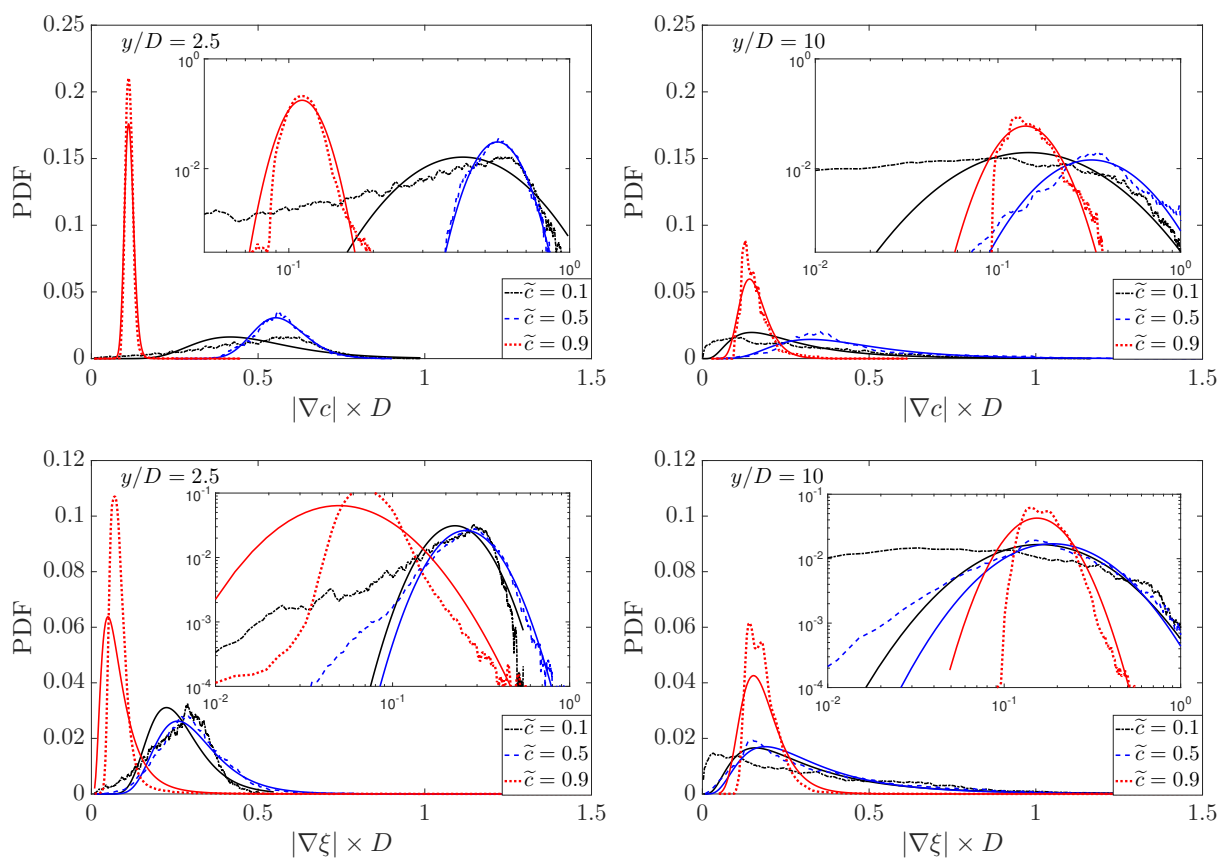

Figure 5: PDFs of $|\nabla c|$ and $|\nabla \xi|$ at different locations within the flame. Solid lines represent log-normal approximations.

\subsection{Behaviour and modelling of the Joint PDF of $|\nabla c|$ and $|\nabla \xi|$}

An understanding of the relative behaviour of $|\nabla c|$ and $|\nabla \xi|$ is needed to gain an insight into the physics of the cross scalar dissipation rate of $c$ and $\xi$. In this spirit the direction cosine between $\nabla c$ and $\nabla \xi$ has been calculated at different $y / D$ locations (not shown here) and it is found that $\nabla c$ and $\nabla \xi$ remain collinearly aligned throughout the flame. This alignment can exist due to the lower Reynolds number of the jet and needs to be investigated further for different flow conditions. The relative behaviour of $\nabla c$ and $\nabla \xi$ is further investigated by analysing the joint $\mathrm{PDF}$ of $|\nabla c|$ and $|\nabla \xi|$ and the results are shown in Figs. 6 and 7 (exemplary for $\widetilde{c}=0.5$ at two locations downstream of the jet exit). Note that all the values of $\widetilde{c}$ and axial locations 
show a qualitatively similar behaviour for the joint $\mathrm{PDF}$ of $|\nabla c|$ and $|\nabla \xi|$. It has been found that $|\nabla c|$ and $|\nabla \xi|$ are negatively correlated near the jet exit $(y / D=2.5)$ and this correlation becomes positive as the distance from the jet exit increases as shown in Figs. 6-7. In order to investigate this further, joint PDF of $|\nabla c|$ and $|\nabla \xi|$ is compared with the PDF of $|\nabla c| \times$ PDF of $|\nabla \xi|$ in Figs. 6 and 7 and it has been found that $P(|\nabla c|,|\nabla \xi|)$ and $P(|\nabla c|) \times P(|\nabla \xi|)$ do not match, which implies that $|\nabla c|$ and $|\nabla \xi|$ are not statistically independent.

The joint $\mathrm{PDF}$ of $|\nabla c|$ and $|\nabla \xi|$ can be approximated by a bivariate log-normal distribution, defined as :

$$
f_{\Lambda_{1}, \Lambda_{2}}\left(\mu_{\Lambda_{1}, \Lambda_{2}}, \sigma_{\Lambda_{1}, \Lambda_{2}}\right)=\frac{\exp (-Q / 2)}{2 \pi \Lambda_{1} \Lambda_{2} \sigma_{\Lambda_{1}} \sigma_{\Lambda_{2}} \sqrt{1-p^{2}}}
$$

where $\mu_{\Lambda_{i}}$ and $\sigma_{\Lambda_{i}}$ are the respective means and standard deviations of $\Lambda_{i}, p$ is the Pearson's correlation coefficient defined as $p=E\left[\left(\ln \left(\Lambda_{1}\right)-\mu_{\Lambda_{1}}\right)\left(\ln \left(\Lambda_{2}\right)-\right.\right.$ $\left.\left.\mu_{\Lambda_{2}}\right)\right] / \sigma_{\Lambda_{1}} \sigma_{\Lambda_{2}}$, where $E(x)$ is the expected value of $x$. In Eq. (7) $Q$ is the exponential coefficient :

$$
\begin{aligned}
Q=\frac{1}{1-p^{2}} & {\left[\left(\frac{\ln \left(\Lambda_{1}\right)-\mu_{\Lambda 1}}{\sigma_{\Lambda_{1}}}\right)^{2}+\left(\frac{\ln \left(\Lambda_{2}\right)-\mu_{\Lambda_{2}}}{\sigma_{\Lambda_{2}}}\right)^{2}\right.} \\
& \left.-2 p\left(\frac{\ln \left(\Lambda_{1}\right)-\mu_{\Lambda_{1}}}{\sigma_{\Lambda_{1}}}\right)\left(\frac{\ln \left(\Lambda_{2}\right)-\mu_{\Lambda_{2}}}{\sigma_{\Lambda_{2}}}\right)\right] .
\end{aligned}
$$

In the case when $p=0$ in Eq. (7)-(8), one obtains $f_{\Lambda_{1}, \Lambda_{2}}=f_{\Lambda_{1}} f_{\Lambda_{2}}$. Figures 6-7 show the values of $f_{c, \xi}$ using the correlation coefficient extracted from the DNS data and $f_{c, \xi}$ with $p=0$ (i.e. no correlation). The expression in Eq. 7 captures the qualitative behaviour of the joint PDF of $|\nabla c|$ and $|\nabla \xi|$ with $p \neq 0$, whereas the prediction from the bivariate log-normal distribution with $p=0$ does not compare well with the DNS data. It needs to be emphasised 
that the bivariate log-normal distribution captures the qualitative behaviour of the joint PDF of $|\nabla c|$ and $|\nabla \xi|$ extracted from the DNS data and serves as a first approximation to guide the modelling effort.
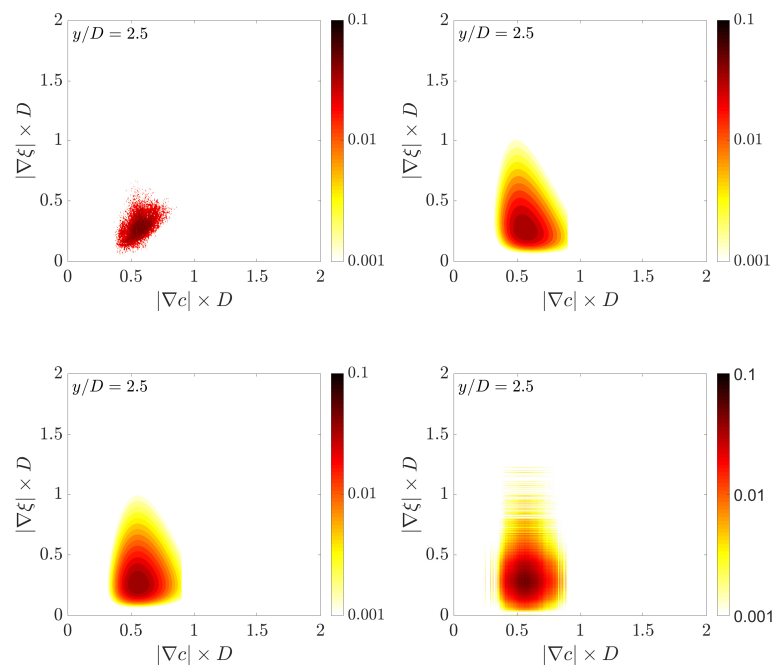

Figure 6: Joint PDF of $|\nabla c|$ and $|\nabla \xi|$ (top left), log-normal PDF with correlation $p=$ -0.2201 (top right), log-normal PDF without correlation (bottom left), PDF of $|\nabla c| \times$ PDF of $|\nabla \xi|$ (bottom right) at $\widetilde{c}=0.5$ and $y / D=2.5$.

\section{Conclusions}

The statistics of $c, \xi$ and their gradients $(|\nabla c|$ and $|\nabla \xi|)$ have been investigated utilising a carrier phase DNS dataset of a pulverised coal jet flame [13]. The variance of the progress variable $\left(\widetilde{c^{\prime \prime 2}}\right)$ and mixture fraction $\left(\widetilde{\xi^{\prime \prime}}\right)$ have been found to increase with the height of the flame which is attributed to the increased turbulent mixing and devolatilisation of the coal particles. The covariance $\left(\widetilde{c^{\prime \prime} \xi^{\prime \prime}}\right)$ also increases in magnitude in the middle of the flame, whereas it assumes negative values towards the burnt side of the flame. The 

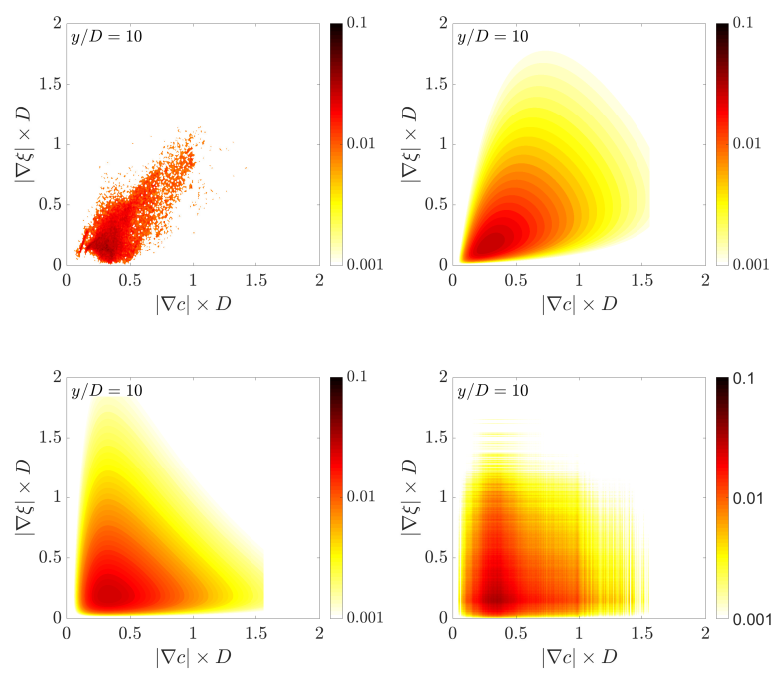

Figure 7: Joint PDF of $|\nabla c|$ and $|\nabla \xi|$ (top left), log-normal PDF with correlation $p=$ 0.5385 (top right), log-normal PDF without correlation (bottom left), $\mathrm{PDF}$ of $|\nabla c| \times \mathrm{PDF}$ of $|\nabla \xi|$ (bottom right) at $\widetilde{c}=0.5$ and $y / D=10$.

aforementioned behaviours have been explained in terms of turbulent mixing and devolatilisation of the coal particles.

It has been found that the Favre PDFs of $c$ and $\xi$ are well represented by the standard $\beta$-function both in terms of the shape and magnitude across different locations and different values of $\widetilde{c}$. Furthermore, it has been observed that the log-normal distribution can accurately represent the PDFs of $|\nabla \xi|$ and $|\nabla c|$ with negligible departures from the DNS data at different values of $\widetilde{c}$. This is consistent with the previous experimental and numerical findings for gaseous phase combustion $[40,41]$. Finally, the joint PDF of $|\nabla c|$ and $|\nabla \xi|$ has been compared with the product of PDF of $|\nabla c|$ and PDF of $|\nabla \xi|$ extracted from DNS and it has been found that $|\nabla c|$ and $|\nabla \xi|$ are not statistically independent for the case investigated. The bivariate log-normal 
PDFs with and without correlation have also been considered, and it has been found that the presence of correlation leads to an improved agreement with the DNS data although some discrepancies could still be observed due to the difficulties in approximation for PDF of $|\nabla c|$ and $\mathrm{PDF}$ of $|\nabla \xi|$ with the log-normal distribution.

Further experimental and DNS investigations of the quantities studied in this work are needed under different flow conditions (i.e. higher Reynolds numbers and different flow configurations $[44,45])$ to improve RANS and LES closures for accurate modelling of pulverised coal combustion.

\section{Acknowledgements}

The authors would like to acknowledge the financial support from EPSRC, British Council and JSPS. This research was partially supported by MEXT (Ministry of Education, Culture, Sports, Science, and Technology) as "Priority issue on Post-K computer" (Accelerated Development of Innovative Clean Energy Systems).

\section{References}

[1] R. Kurose, H. Makino, A. Suzuki, Fuel 83 (2004) 693-703.

[2] B. Franchetti, F. Cavallo Marincola, S. Navarro-Martinez, A. Kempf, Proc. Combust. Inst. 34 (2013) 2419-2426.

[3] B. Franchetti, F. Cavallo Marincola, S. Navarro-Martinez, A. Kempf, Fuel 181 (2016) 491-502.

[4] M. Rieth, A. Clements, M. Rabaçal, F. Proch, O. T. Stein, A. Kempf, Proc. Combust. Inst. 36 (2017) 2181-2189. 
[5] M. Rieth, F. Proch, A. Clements, M. Rabaçal, A. Kempf, Proc. Combust. Inst. 36 (2017) 3371-3379.

[6] X. Wen, K. Luo, H. Jin, J. Fan, Combust. Theory Model. 21 (2017) 925-953.

[7] S. P. Malkeson, N. Chakraborty, Flow, Turbul. Combust. 86 (2010) 1-44.

[8] S. P. Malkeson, S. Ruan, N. Chakraborty, N. Swaminathan, Combust. Sci. Technol. 185 (2013) 1329-1359.

[9] D. H. Wacks, N. Chakraborty, Combust. Sci. Technol. 188 (2016) 21492177.

[10] X. Wen, K. Luo, Y. Luo, H. I. Kassem, H. Jin, J. Fan, Appl. Energy 183 (2016) 1086-1097.

[11] C. D. Pierce, P. Moin, J. Fluid Mech. 504 (2004) 73-97.

[12] M. Ihme, C. M. Cha, H. Pitsch, Proc. Combust. Inst. 30 (2005) 793-800.

[13] T. Hara, M. Muto, T. Kitano, R. Kurose, S. Komori, Combust. Flame 162 (2015) 4391-4407.

[14] S. M. Hwang, R. Kurose, F. Akamatsu, H. Tsuji, H. Makino, M. Katsuki, Energy and Fuels 19 (2005) 382-392.

[15] B. Franzelli, E. Riber, M. Sanjosé, T. J. Poinsot, Combust. Flame 157 (2010) 1364-1373.

[16] A. L. Pillai, R. Kurose, Appl. Acoust. 133 (2018) 16-27. 
[17] T. Kitano, K. Kaneko, R. Kurose, S. Komori, Combust. Flame 170 (2016) 63-78.

[18] Y. Hu, R. Kurose, Combust. Flame 188 (2018) 227-242.

[19] D. Messig, M. Vascellari, C. Hasse, Combust. Theory Model. 21 (2017) $700-721$.

[20] X. Wen, H. Wang, Y. Luo, K. Luo, J. Fan, Phys. Fluids 29 (2017) 083607.

[21] R. Bilger, in: P. Libby, F. Williams (Eds.), Turbulent Reacting Flows, volume 44 of Topics in Applied Physics, Springer, 1980, pp. 65-113.

[22] K. N. C. Bray, J. B. Moss, Acta Astronaut. 4 (1977) 291-319.

[23] N. Chakraborty, J. W. Rogerson, N. Swaminathan, Phys. Fluids 20 (2008) 045106.

[24] G. Ribert, M. Champion, P. Plion, Combust. Sci. Technol. 176 (2004) 907-923.

[25] P. A. Libby, F. A. Williams, Combust. Sci. Technol. 161 (2000) 351-390.

[26] H. Kolla, J. W. Rogerson, N. Chakraborty, N. Swaminathan, Combust. Sci. Technol. 181 (2009) 518-535.

[27] Y. Gao, N. Chakraborty, N. Swaminathan, Combust. Sci. Technol. 186 (2014) 1309-1337.

[28] N. Chakraborty, M. Katragadda, R. S. Cant, Flow, Turbul. Combust. 87 (2010) 205-235. 
[29] U. Ahmed, R. Prosser, A. J. Revell, Flow, Turbul. Combust. 93 (2014) 637-663.

[30] D. Butz, Y. Gao, A. Kempf, N. Chakraborty, Combust. Flame 162 (2015) 3180-3196.

[31] T. Ma, O. T. Stein, N. Chakraborty, A. Kempf, Combust. Theory Model. 17 (2013) 431-482.

[32] T. Ma, O. T. Stein, N. Chakraborty, A. Kempf, Combust. Theory Model. 18 (2014) 32-64.

[33] U. Ahmed, R. Prosser, Combust. Theory Model. 20 (2016) 34-57.

[34] U. Ahmed, R. Prosser, Flow, Turbul. Combust. 100 (2018) 39-73.

[35] N. Swaminathan, K. N. C. Bray, Combust. Flame 143 (2005) 549-565.

[36] S. Fedotov, M. Ihme, H. Pitsch, Phys. Rev. E 71 (2005) 1-8.

[37] H.-W. Ge, E. Gutheil, Atom. and Sprays 16 (2006) 531-542.

[38] E. Gutheil, Handbook of combustion, Wiley-VCH Verlag GmbH \& Co, KGaA.

[39] P. Jenny, D. Roekaerts, N. Beishuizen, Prog. Energy Combust. Sci. 38 (2012) 846-887.

[40] D. Geyer, A. Kempf, A. Dreizler, J. Janicka, Proc. Combust. Inst. 30 (2005) 681-689.

[41] C. N. Markides, N. Chakraborty, Chem. Eng. Sci. 90 (2013) 221-241. 
[42] C. Pantano, J. Fluid Mech. 514 (2004) 231-270.

[43] R. Knaus, J. Oefelein, C. Pantano, Flow, Turbul. Combust. 89 (2012) $37-71$.

[44] K. Luo, H. Wang, J. Fan, F. Yi, Energy and Fuels 26 (2012) 6128-6136.

[45] Y. Luo, X. Wen, H. Wang, K. Luo, H. Jin, J. Fan, Combust. Theory Model. 7830 (2018) 1-26. 